\title{
GUIDE EXPANSIONS FOR THE RECURSIVE PARAMETRIC SOLUTION OF POLYNOMIAL DYNAMICAL SYSTEMS
}

\author{
G. F. D. DUFF', R. B. LEIPNIK ${ }^{2}$ and C. E. M. PEARCE ${ }^{3}$
}

(Received November 15, 2004)

\begin{abstract}
Recursive parametric series solutions are developed for polynomial ODE systems, based on expanding the system components in series of a form studied by Weiss. Individual terms involve first-order driven linear ODE systems with variable coefficients. We consider Lotka-Volterra systems as an example.
\end{abstract}

2000 Mathematics subject classification: $34 \mathrm{C} 20,35 \mathrm{C} 10$.

Keywords and phrases: guide expansions, general series solutions, core functions.

\section{Introduction}

The method developed here is inspired by Hopf's famous study [6] of Burger's equation (see also [2]). It can be viewed as a generalised Lagrange expansion that is adaptable to the ODE system of interest. For the Navier-Stokes PDE application [8] an infinite series of reaction-diffusion operators appears. There are also echoes of Poincaré's stretched-time perturbations and psi-series $[10,12]$. The objective is to obtain solutions to nonlinear vector operator equations as a sum of solutions of a series of recursivelydefined linear operators with variable weights while preserving for exploitation the coefficients of the original vector operator in the various linear operators. This allows arbitrary orders of variable perturbations. The method has application in connection with both exact and numerical solutions. We present a streamlined and self-contained account of the basic underlying ideas. Detailed applications will appear elsewhere (see [4]).

\footnotetext{
'deceased, formerly of the Department of Mathematics, University of Toronto.

${ }^{2}$ Mathematics Department, University of California, Santa Barbara, CA 93106-3080, USA; e-mail: leipnik@math.ucsb.edu.

${ }^{3}$ School of Mathematical Sciences, The University of Adelaide, Adelaide SA 5005, Australia; e-mail: charles.pearce@adelaide.edu.au.

(C) Australian Mathematical Society 2006, Serial-fee code 1446-1811/06
} 
We consider an explicit polynomial ODE system of the form

$$
\dot{x}_{j}(t)=a_{j}(t)+\sum_{m=1}^{N} \sum_{k_{1}, \ldots, k_{m}=1}^{n} a_{j: k_{1} \ldots k_{m}} x_{k_{1}} \cdots x_{k_{m}} \quad(j=1, \ldots, n),
$$

where the order zero coefficients (drivers) $a_{j}$ and the higher-order coefficients are differentiable on a set $S$. Here $N$ may exceed $n$. By Peano's theorem, continuous solutions exist. Uniqueness theorems require dominance conditions, such as those of Lipschitz or Osgood [9]. Linear systems and matrix Riccati systems have known explicit solutions [7]. Currently vector Riccati systems, and in particular LotkaVolterra species-dynamical solutions do not, except when all the coefficients are given in terms of Taylor series convergent in a region $T=\left\{t:\left|t-t_{0}\right|<r\right\}$. In this case the solutions are in principle given by explicit convergent Taylor series on $T$ using convolution algebra. When the solutions are of nonpolynomial form, such as $\exp \left(t^{2}\right)$ or $\ln |\ln t|$, Taylor series may be impractical and other expressions are desirable.

The analysis turns on the use of a differentiable core function $h(t)$ which is entirely at our disposal and could be, for example, a suitably-stretched time, a key ingredient in the $x_{j}(t)$, or a combination of these. The underlying idea is to express the $x_{j}(t)$ as power series in $1 / h(t)$ with variable coefficients. The strategy in a given application is to choose the core function to reduce the structural complexity of the problem or speed the convergence of various series involved.

The choice of $h$ allows some intuition or information about system behaviour to be incorporated. The method can be extended to PDEs, where indeed it was first used to produce exact solutions. Computationally an extensive memory is required compared to traditional Runge-Kutta/Adams computing for ODEs to accommodate the recursive tail. The Picard method of recursive integration to provide existence proofs has not been computationally successful. This work may be regarded as an adaptation of Picard's original idea using Hopf's algebraic approach.

There are two physically distinguishable cases. In the first, $a_{j}(t)=0$ for all $j$, so the dynamics are free. In case II, with core-dependent drivers, the $a_{j}(t)$ are expressible in terms of the core function via the series

$$
a_{j}(t)=\sum_{p=1}^{\infty} b_{j . p}(t) h^{-p}
$$

assumed convergent on a set $T$. Similarly we ascribe to the $x_{j}(t)$ a decomposition

$$
x_{j}(t)=\sum_{p=1}^{\infty} u_{j, p}(t) h^{-p} \quad(j=1, \ldots, n) .
$$

The $u_{j, p}$ are termed guides and (1.3) a guide expansion. 
By scaling $b_{j . p}$ by a suitable power of $h$ we obtain an altemative parameterisation of the form

$$
a_{j}(t)=\sum_{p=0}^{\infty} b_{j, p}(t)[h(t)]^{-p}
$$

in which (for at least one value of $j$ ) $b_{j .0}(t)$ is not identically zero. We associate with this the formal expansion

$$
x_{j}(t)=\sum_{p=0}^{\infty} u_{j . p}(t) h^{-p} \quad(j=1, \ldots, n) .
$$

This formulation with non-core drivers is sometimes more appropriate, though a treatment as it stands requires a significant extension of the procedures that suffice for cases I and II. For brevity we refer to it as case III.

With $1 / h=\sigma,(1.3)$ is a special ODE case (for $\alpha=0$ ) of the Weiss series $\sigma^{-\alpha} \sum_{n=0}^{\infty} \phi_{n} \sigma^{n}$ used in connection with the Painlevé property [13]. See also [1, 3, 11].

Formally, a classic example of a case III-type expansion with constant guides is provided by the Lagrange formula

$$
f(\zeta)=f(a)+\sum_{p=1}^{\infty} \frac{1}{p !}\left[\frac{d^{p-1}}{d a^{p-1}}\left\{f^{\prime}(a)[\phi(a)]^{p}\right\}\right] w^{p}
$$

(see [14, pages 132-133]).

In Section 2 we present the recursive differential algebra required for cases I and II and in Section 3 that for case III. A discrete Hilbert-space construction of guides for a given core $h$ and target functions $x_{j}$ is outlined in Section 4. In Section 5 we give a discussion of the method for Lotka-Volterra systems.

\section{Case I and II numerator recursions}

From (1.3) we have formally that

$$
\dot{x}_{j}=\sum_{p=1}^{\infty}\left[\dot{u}_{j, p}-(p-1) \dot{h} u_{j . p-1}\right] h^{-p}
$$

Substitution into (1.1) yields for $j=1, \ldots, n$ that the right-hand side of $(2.1)$ is equal to

$$
a_{j}(t)+\sum_{m=1}^{N} \sum_{k_{1} \ldots, k_{m}=1}^{n} a_{j: k_{1} \ldots . . k_{m}} \prod_{j=1}^{m} \sum_{p=1}^{\infty} u_{k_{j}, p} h^{-p}
$$


so that

$$
\begin{aligned}
\sum_{p=1}^{\infty}\left[\dot{u}_{j, p}-(p-1) \dot{h} u_{j, p-1}\right] h^{-p} \\
\quad=\sum_{p=1}^{\infty} b_{j, p} h^{-p}+\sum_{m=1}^{N} \sum_{k_{1}, \ldots, k_{m}=1}^{n} a_{j ; k_{1}, \ldots, k_{m}} \prod_{j=1}^{m} \sum_{p=1}^{\infty} u_{k_{j}, p} h^{-p} .
\end{aligned}
$$

Put $\boldsymbol{u}_{k}:=\left(u_{k, 0}, u_{k, 1}, \ldots\right)$ and $\left(\boldsymbol{u}_{k}\right)_{p}:=u_{k, p}$. We introduce the convolution

$$
\left(\boldsymbol{u}_{k} \star \boldsymbol{u}_{\ell}\right)_{p}=\sum_{r=0}^{p} u_{k, r} u_{\ell, p-r}
$$

In the present context

$$
u_{k, 0}=0 \text { for } k=1, \ldots, n \text {, }
$$

so the right-hand side of (2.4) reduces to $\sum_{r=1}^{p-1} u_{k, r} u_{\ell, p-r}$. We write

$$
\underset{r=1}{\star} u_{k_{r}}=u_{k_{1}} \star u_{k_{2}} \star \cdots \star u_{k_{m}}
$$

for the $(m-1)$-fold convolution and interpret $\star_{r=1}^{\prime} \boldsymbol{u}_{k_{r}}=\boldsymbol{u}_{k_{1}}$.

Complete separation of (2.3) according to the exponent of $h$ yields

$$
\dot{u}_{j .1}=b_{j .1}+\sum_{k=1}^{n} a_{j: k} u_{k, 1} \quad(j=1, \ldots, n)
$$

and for $p>1$

$$
\dot{u}_{j, p}=(p-1) \dot{h} u_{j, p-1}+b_{j, p}+\sum_{m=1}^{N} \sum_{k_{1}, \ldots, k_{m}=1}^{n} a_{j ; k_{1} \ldots . k_{m}}\left(\stackrel{m}{\star} u_{r=1}\right)_{p} .
$$

By (2.5) the right-hand side of (2.7) involves terms $u_{k, \ell}(k=1, \ldots, n)$ only for $\ell<p$, so that in principle (2.6) may be solved for the $u_{j .1}$ and then (2.7) solved for $u_{j, p}$ $(j=1, \ldots, n)$ recursively in $p$.

In practice $u_{j, p}$ may diverge for $p \rightarrow \infty$ while the basic components $v_{j, p}(t)=$ $u_{j . p}(t)[h(t)]^{-p}$ of $x_{j}$ are generally better behaved. We have immediately the recursions

$$
\frac{d}{d t}\left(v_{j, 1} h\right)=b_{j, 1}+\sum_{k=1}^{n} a_{j: k} v_{k, 1} h
$$

and for $p>1$

$$
\frac{d}{d t}\left(v_{j, p} h^{r}\right)-(p-1) \dot{h} v_{j . p-1} h^{\rho-1}=b_{j . p}+h^{p} \sum_{m=1}^{N} a_{j ; k_{1} \ldots . . k_{m}}\left(\underset{r=1}{\stackrel{m}{\star} v_{k_{r}}}\right)_{p}
$$


If $c_{j . p}=b_{j . p} h^{-p}$ denotes the $p$-th term in the expansion of $a_{j}$, these recursions become

$$
\dot{v}_{j, 1}+v_{j, 1} \frac{\dot{h}}{h}=c_{j .1}+\sum_{k=1}^{n} a_{j ; k} v_{k, 1}
$$

and for $p>1$

$$
\dot{v}_{j, p}+\frac{\dot{h}}{h}\left[p v_{j, p}-(p-1) v_{j, p-1}\right]=c_{j, p}+\sum_{m=1}^{N} \sum_{k_{1}, \ldots, k_{m}=1}^{n} a_{j ; k_{1} \ldots, k_{m}}\left(\underset{r=1}{\star m} v_{k_{r}}\right)_{p} .
$$

This is a feedback system: if $v_{j, p}$ increases rapidly in $t$, a reduction is induced in its successor $v_{j, p+1}$ when $\dot{h}$ is positive. As far as convergence is concerned, the choice of the feedback coefficient $\dot{h} / h$ is crucial in this recursive system. Experimentation with Lotka-Volterra systems (for which $N=2$ ) indicates that small $\dot{h} / h$ can lead to fast convergence for small $t$ followed by unstable oscillations. Large $\dot{h} / h$ promotes global stability of the successive approximants $\sum_{p=1}^{\ell} v_{j, p}(t)$ but slow convergence compared to intermediate values, for which both convergence and stability are possible. This suggests the use of time-dependent $\dot{h} / h$, but a suitable constant choice for $\dot{h} / h$ seems to often suffice when $a_{j: k}$ and $a_{j: k, \ell}$ are constant.

For the solution and/or numerical evaluation of the $x_{j}(t)$, we employ

$$
x_{j}(t)=\sum_{p=1}^{\infty} v_{j, p}(t) \text {. }
$$

This method preserves the full set of parameters in the original system at the cost of maintaining sequential convolutions containing all parameters. Each equation contributes a matrix with $(j, k)$ entry $a_{j: k}-k(\dot{h} / h) \delta_{j, k}$ in a linear system driven by the driving coefficients $a_{j}$ and a nonlinear combination of preceding states.

\section{Extension to non-core drivers}

We now consider the solution of (1.1) using the expansions (1.4) and (1.5). In place of (2.3) we have

$$
\begin{aligned}
\dot{u}_{j, 0} & +\sum_{p=1}^{\infty}\left[\dot{u}_{j, p}-(p-1) \dot{h} u_{j, p-1}\right] h^{-p} \\
& =\sum_{p=0}^{\infty} b_{j, p} h^{-p}+\sum_{m=1}^{N} \sum_{k_{1}, \ldots, k_{m}=1}^{n} a_{j: k_{1} \ldots . . k_{m}} \prod_{j=1}^{m} \sum_{p=1}^{\infty} u_{k_{j}, p} h^{-p} .
\end{aligned}
$$

For convenience, we introduce $u_{0}=\left(u_{1.0}, \ldots, u_{n, 0}\right)$ and

$$
\Phi_{j}\left(u_{0}\right)=\sum_{m=1}^{N} \sum_{k_{1}, \ldots . k_{m}=1}^{n} a_{j: k_{1}, \ldots, k_{m}} \prod_{j=1}^{m} u_{k_{j}, 0} \quad(j=1, \ldots, n) .
$$


Equating coefficients of $h^{-p}$ in (3.1) for $p=0$ now gives the nonlinear system

$$
\dot{u}_{j, 0}=b_{j, 0}+\Phi_{j}\left(u_{0}\right) \quad(j=1, \ldots, n) .
$$

Two possibilities arise, according to whether or not we have a solution $u_{0}$ to (3.2).

Suppose first that $u_{0}$ satisfies (3.2). Equating coefficients of $h^{-p}$ in (2.4) for $p>0$ yields (2.7). The convolution term $\left(u_{k_{1}} \star \cdots \star u_{k_{m}}\right)_{p}$ contains the $n$ terms $u_{j, p} \prod_{k \neq j} u_{k, 0}$. All other terms in the convolution are independent of $u_{j, p}$, involving products of quantities $u_{k, q}$ with $q<p$. Hence the equations for $p>0$ form a system of driven linear ODEs

$$
\dot{u}_{j, p}=b_{j, p}+\sum_{m=1}^{N} \sum_{k_{i}, \ldots . k_{m}=1}^{n} a_{j: k_{1} \ldots, k_{m}}^{(p)} u_{k_{m}, p}+F_{j, p}\left(u_{k, r} ; k=1, \ldots, n, r<p\right),
$$

where the $a^{(p)}$ terms are combinations of the coefficients with products of components of $\boldsymbol{u}_{0}$ and the $F_{j, p}$ are nonlinear in the $u_{k, r}$. The general solution for linear systems with variable coefficients and continuous driving functions is addressed in Yoshida [15]. See also [5].

If instead $\boldsymbol{u}_{0}$ does not satisfy (3.2), we combine the zeroth and first-order conditions as

$$
\left.\dot{u}_{j, 0}+\dot{u}_{j, 1} h^{-1}=b_{j, 0}+b_{j, 1} h^{-1}+\Phi_{j}\left(u_{0}\right)+h^{-1} \sum_{m=1}^{N} \sum_{k_{1} \ldots . . k_{m}=1}^{n} a_{j, k_{1} \ldots, k_{m}}\left(\stackrel{m}{\star} u_{r=1}\right)_{k_{r}}\right)_{1} .
$$

We define

$$
\Delta_{j, 0}\left(u_{0}\right)=h(t)\left[\dot{u}_{j .0}-\left(b_{j .0}+\Phi_{j}\left(u_{0}\right)\right)\right] \quad(j=1, \ldots, n),
$$

which we may regard as an " $h$-magnified error" arising from non-satisfaction of the zeroth-order condition. Then (3.3) may be recast for $j=1, \ldots, n$ as

$$
\dot{u}_{j .1}=b_{j .1}-\Delta_{j .0}+\sum_{m=1}^{N} \sum_{k_{1} \ldots, k_{m}=1}^{n} a_{j ; k_{1}, \ldots, k_{m}}\left(\stackrel{m}{\star} \boldsymbol{u}_{k_{r}}\right)_{1}
$$

Since

$$
\left(\underset{t=1}{\star} u_{k_{r}}\right)_{1}=\left(\prod_{j=1}^{m} u_{k_{j} .0}\right) \sum_{\ell=1}^{m} \frac{u_{\ell .1}}{u_{\ell .0}}
$$

(3.4) is a linear differential equation for $u_{j, 1}$ with coefficients depending only on $\boldsymbol{u}_{0}$. Thus (3.4) permits explicit solution. The higher-order equations (2.7) for $p>1$ and $j=1, \ldots, n$ may be treated similarly. The convolution has $n$ terms of the form $u_{j, p} \prod_{k \neq j} u_{k, 0}$ linear in $u_{j, p}$. 
As before, these equations can be written in terms of the $v_{j, p}$. In particular, $u_{j .0}=v_{j .0}$ and

$$
\begin{aligned}
\dot{v}_{j .1}= & c_{j .1}-\left[\dot{v}_{j, 0}-\left(b_{j, 0}+\Phi_{j}\left(v_{0}\right)\right)\right] \\
& +\sum_{k=1}^{n}\left(a_{j: k}-\frac{\dot{h}}{h} \delta_{j, k}\right) v_{k, 1}+\sum_{m=2}^{N} \sum_{k_{1}, \ldots, k_{m}=1}^{n} a_{j: k_{1} \ldots . . k_{m}}\left(\underset{r=1}{\star} v_{k_{r}}\right)_{1} .
\end{aligned}
$$

\section{Construction of the guide coefficients}

A discrete Hilbert-space technique is sufficient to construct the guide coefficients for a given $x, h$ and interval $S$. Let $\left\{\psi_{k, n}(t)\right\}$ be the (essentially unique) double sequence of functions on $S$ defined for $k, \ell, n=0,1,2, \ldots$ by

$$
\int_{S} \psi_{k, n}(t) \psi_{\ell, n}(t) h^{-n}(t) d t=\delta_{k, \ell}
$$

For $S$ finite and $n=0$, the $\psi_{k .0}(t)$ are Legendre polynomials, while for $S$ semi-infinite and $n=0$ they are Laguerre functions. For $S$ infinite and $n=0$, they are Hermite functions. If $h$ is exponential and $S$ semi-infinite, the $\psi_{k, n}(t)$ are $n$-scaled Laguerre polynomials.

Also form the quadruple sequence

and define

$$
\chi_{k, n: \ell, r}=\int_{S} \psi_{k, n}(t) \psi_{\ell, r}(t) h^{-n}(t) d t
$$

$$
c_{\ell, r}=\int_{S} \psi_{\ell, r}(t) x(t) d t, \quad \phi_{n}(t)=\sum_{k=0}^{\infty} a_{k, n} \psi_{k, n}(t) .
$$

Our intention is to choose $a_{k, n}$ to produce $x(t)=\sum_{n=0}^{\infty} \phi_{n}(t) h^{-n}(t)$. This would imply that

$$
\begin{aligned}
c_{\ell, r} & =\int_{S} \psi_{\ell, r}(t) \sum_{n=0}^{\infty} \phi_{n}(t) h^{-n}(t) d t \\
& =\int_{S} \psi_{\ell, r}(t) \sum_{n, k=0}^{\infty} a_{k, n} \psi_{k, n}(t) h^{-n}(t) d t \\
& =\sum_{n, k=0}^{\infty} a_{k, n} \psi_{k, n}(t) h^{-n}(t) d t=\sum_{n, k=0}^{\infty} a_{k, n} \chi_{k, n: \ell, r}
\end{aligned}
$$

if the various sums are absolutely convergent. 
The last relation is a doubly-infinite set of equations for $\left\{a_{k, n}\right\}$ in terms of $\left\{c_{\ell, r}\right\}$. Let $\left\{\chi_{\ell, r}^{k_{1}, n_{1}}\right\}$ be defined implicitly for each $k, k_{1}, n, n_{1}$ by the inverse sequence condition

$$
\sum_{\ell, r=0}^{\infty} \chi_{k, n: \ell, r} \chi_{\ell, r}^{k_{1, n}}=\delta_{k, k_{1}} \delta_{n, n_{1}}
$$

Then

$$
\begin{aligned}
\sum_{\ell, r=0}^{\infty} c_{\ell, r} \chi_{\ell, r}^{k_{1}, n_{1}} & =\sum_{\ell, r=0}^{\infty} \sum_{n, k=0}^{\infty} a_{k, n} \chi_{k, n ; \ell, r} \chi_{\ell, r}^{k_{1}, n_{1}} \\
& =\sum_{n, k=0}^{\infty} a_{k, n} \sum_{\ell, r=0}^{\infty} \chi_{k, n, \ell, r} \chi_{\ell, r}^{k_{1}, n_{1}} \\
& =\sum_{n, k=0}^{\infty} a_{k, n} \delta_{k, k_{1}} \delta_{n, n_{\ell}}=a_{k_{1}, n_{1}},
\end{aligned}
$$

so that the coefficients $a_{k, n}$ and $\phi_{n}(t)$ are determined. If the sequences $\left\{\psi_{k, n}\right\}$ are complete for each $n$, then the above calculation is reversible, determining $x(t)$ via $\left\{\chi_{k, n: \ell, r}\right\}$ and its inverse. The orthogonal examples given above are all complete. We note that $\chi_{k, n: \ell, n}=\delta_{k, \ell}$ for each $n$.

\section{Riccati system application}

An example of a class of nonlinear system amenable to the method is the driven $q$-dimensional Riccati system of Lotka-Volterra type

$$
\dot{x}_{j}=e_{j}(t)+\sum_{i=1}^{q} a_{j: i} x_{i}-\sum_{\ell=1}^{q} a_{j \ell \ell, \ell} x_{\ell}^{2}+\sum_{\ell \neq k=1}^{q} a_{j: \ell, k} x_{\ell} x_{k} \quad(j=1, \ldots, q)
$$

with $a_{j: \ell \ell \ell} \geq 0$.

Let $s_{1}, \ldots, s_{q}$ denote the singular values of the matrix $A=\left(a_{j ; i}\right)$ and $s=\max _{k} s_{k}$. We take

$$
h(t)=e^{(s+\delta) t}
$$

for $\delta>0$ small. We may use the expansion

$$
e_{j}=\sum_{p=1}^{\infty} b_{j . p} h^{-p}
$$

given by $b_{j . p}=e_{j}(t) h(t) \delta_{p, 1} t$, so that $c_{j, p}=e_{j}(t) \delta_{p, 1}$. 
Here $\dot{h} / h=s+\delta$ is constant and the guide recursion system is the sequence of first-order linear equations

$$
\begin{aligned}
\dot{v}_{j, 1}= & e_{j}-(s+\delta) v_{j, 1}+\sum_{\ell=1}^{q} a_{j, \ell} v_{\ell, 1} \quad \text { for } j=1, \ldots, q \\
\dot{v}_{j, p}= & (s+\delta)\left[(p-1) v_{j, p-1}-p v_{j, p}\right]+\sum_{i=1}^{q} a_{j ; i} v_{i, p}-\sum_{\ell=1}^{q} a_{j, \ell, \ell}\left(v_{\ell} \star v_{\ell}\right)_{p} \\
& +\sum_{\ell \neq k=1}^{q} a_{j: \ell, k}\left(v_{\ell} \star v_{\ell}\right)_{p} \quad \text { for } p>1 \text { and } j=1, \ldots, q .
\end{aligned}
$$

If the initial conditions are

$$
v_{j, p}\left(t_{0}\right)=x_{j, 0} \delta_{p, 1} \quad \text { for } p \geq 1 \text { and } j=1, \ldots, q \text {, }
$$

then $x_{j}\left(t_{0}\right)=x_{j, 0}$.

The use of the enhanced singular value $s+\delta$ guarantees that

$$
\left|v_{j, 1}(t)-\int_{t_{0}}^{t} e_{j}(\tau) d \tau\right| \rightarrow 0 \quad \text { exponentially as } t \rightarrow \infty \text {. }
$$

The equations for $v_{j, p}$ retain the system structure, with a negative feedback for stabilisation. The procedure is suitable for computer programming and replaces in an orderly way a nonlinear system by a similarly-structured linear system without compromising the former.

\section{References}

[1] F. Cariello and M. Tabor, "Singularities and symmetries in nonlinear evolution equations", in Painlevé Transcendents (eds. D. Levi and P. Winternitz), (Plenum Press, New York, 1992) 315343.

[2] J. D. Cole, "On a quasi-linear parabolic equation", Quart. J. Appl. Math. 9 (1951) 225-236.

[3] G. F. D. Duff, "Limit cycles and rotated vector fields", Ann. Math. 57 (1953) 15-31.

[4] G. F. D. Duff and R. B. Leipnik, "Explicit general series solution for Euler and Navier-Stokes equations", Int. J. Pure Appl. Math. 24 (2005) 211-263.

[5] P. Hartman, Ordinary Differential Equations (Wiley, New York, 1964).

[6] E. Hopf, "The partial differential equation $u_{t}+u u_{x}=\mu u_{x x}$ ", Comm. Pure Appl. Mech. 3 (1950) 20I-230.

[7] R. B. Leipnik, "A canonical form and solution for the matrix Riccati differential equation", $J$. Austral. Math. Soc. Ser. B 26 (1985) 355-361.

[8] R. B. Leipnik, "Exact solutions of the Navier-Stokes equation by recursive series of diffusive quotients", C. R. Math. Rep. Acad. Sci. Canada 18 (1996) $211-216$. 
[9] O. Osgood, "Beweis der Existenze einer Lösung der Differentialgleichung $d y / d x=f(x, y)$ ohne Hinzunahme der Cauchy-Lipschitzsen Bedingung", Monatschefte Math. Physics 9 (1898) 331-345.

[10] H. Poincaré, "Sur les équations de la dynamique et le problème des trois corps", Acta Mathematica 13 (1890) 1-270.

[11] F. Takens, "Forced oscillations and bifurcations", Comm. Math. Rijksuniversitat Utrecht 3 (1974) 1-59.

[12] R. S. Ward, "Multidimensional integrable systems", in Field Theory, Quantum Gravity and Strings // (eds. H. J. de Vega and N. Sánchez), Lecture Notes in Physics 280, (1986) 106-1 16.

[13] J. Weiss, M. Tabor and G. Carnevale, "The Painlevé property for partial differential equations", J. Math. Phys. 24 (1983) 522-526.

[14] E. T. Whittaker and G. N. Watson, A Course in Modern Analysis (Cambridge University Press, Cambridge, 1952).

[15] K. Yoshida, Lectures on Differential and Integral Equations (Wiley, New York, 1960). 